\title{
On-Farm Phenotypic Characterization of Indigenous Sheep Types in Selale Area, Central Ethiopia \\ Bosenu Abera ${ }^{1}$, Kefelegn Kebede ${ }^{2}$, Solomon Gizaw ${ }^{3}$ and Teka Feyera ${ }^{4 *}$
}

${ }^{1}$ Jigjiga University College of Dry Land Agriculture, Ethiopia

${ }^{2}$ Haramaya University College of Agriculture and Environmental Sciences, Ethiopia

${ }^{3}$ Debre Berhan Agricultural Research Center, Ethiopia

${ }^{4}$ Jigjiga University College of Veterinary Medicine, Ethiopia

\begin{abstract}
The study was conducted to physically characterize indigenous sheep types in Selale area, Debre Libanos and Wuchale districts, Central Ethiopia. A total of 560 mature sheep were sampled randomly for characterization of phenotypic traits. Majority of the ewes and rams in both districts had plain coat color pattern $(58.21 \%)$ followed by patchy (33.33\%). Majority of female and male sheep in the study areas had medium and smooth coat cover. All the sampled sheep population in both districts has characteristics of long fat tailed type. Body weight of female sheep in age group1(OPPI), age group2(1PPI) and age group $3(\geq 2 \mathrm{PPI})$ were $24.3 \pm 0.6 \mathrm{~kg}, 25.8 \pm 0.5 \mathrm{~kg}$ and $28.7 \pm 0.2$ $\mathrm{kg}$, respectively, and the values for males in the same age groups were $25.7 \pm 0.3 \mathrm{~kg}, 31.9 \pm 0.8 \mathrm{~kg}$ and $38.2 \pm 2.0$ $\mathrm{kg}$, respectively. Wuchale sheep $(27.9 \pm 0.20 \mathrm{~kg})$ were comparable with Debre Libanos sheep $(27.6 \pm 0.2 \mathrm{~kg})$. Debre Libanos sheep had significantly higher linear body measurements $(P<0.05)$ than Wuchale sheep population. Sex of the sheep had no significant ( $p>0.05$ ) effect on the body weight, ear length and rump length. The interaction of sex and age group significantly $(p<0.05)$ influenced all linear body measurements except ear length of the sheep. The interaction of age group and location was significant $(p<0.05)$ for all linear body measurements. Heart girth and body length were found to be the most important variables for estimation of body weight in sheep. For any breed improvement program and to boost productivity of indigenous sheep, characterization is the baseline so; this preliminary work could be used to support genetic analyses to determine variation between and within these small populations.
\end{abstract}

Keywords: Characterization; Debre Libanos; Phenotypic traits; Selale; Sheep types; Wuchale

\section{Introduction}

Ethiopia is endowed with huge livestock resources of varied and diversified genetic pools with specific adaptations to a wide range of agro-ecologies. Farm animals as a whole are an integral part of the country's agricultural system and are raised both in the highland and lowland areas. In developing countries, livestock production is mostly subsistence oriented and fulfills multiple functions that contribute more for food security $[1,2]$. The demand for livestock products is increasing due to the growing urban population, while farm areas are shrinking considerably as a result of an increase in the rural population [3].

Ethiopia is home for at least 9 breeds and 14 traditional sheep populations [4] with an estimated 25.9 million heads. Out of which about 73.1 percent are females, and about 26.9 percent are males [5]. Of the total sheep population, 75 percent is found in the highlands where mixed crop-livestock systems dominate, while the remaining 25 per cent of the sheep is found in the lowlands [6]. The main production from indigenous sheep populations in Selale area is meat, skin and manure.For planning of community based breeding strategy as well as setting up a useful sheep development program, the genetic and the phenotypic merit and production system of that particular breed is a must. It has been stressed that identification and characterization of livestock genetic resources and their production environment is vital for long-term genetic improvement and sustained use of available resources [7].

On farm characterization can serve as basis for the sustainable improvement and conservation of indigenous animal genetic resources, and has received increasing attention in determining the variation between and within pure breeds [8]. Thus, more comprehensive information specific to on-farm phenotypic characterization of indigenous sheep breeding should be made available. Hence, this study was attempted to physically characterize indigenous sheep types in Selale area, Central Ethiopia.

\section{Materials and Methods}

\section{Study area}

The study was conducted in Selale area, Debre Libanos and Wuchale district, central Ethiopia. Debre Libanos and Wuchale district are located at $85 \mathrm{~km}$ and $75 \mathrm{~km}$ north of the capital Addis Ababa, respectively. Debre Libanos is located in $38^{\circ} 58^{\prime} 33^{\prime \prime} \mathrm{E}$ longitude and $9^{\circ} 63^{\prime} 75^{\prime \prime} \mathrm{N}$ latitude with altitude ranging from 1500 to 2700 m.a.s.l. For Debre Libanos the maximum and minimum annual temperature is $23^{\circ} \mathrm{C}$ and $15^{\circ} \mathrm{C}$, respectively. Its main rainy season occurs between May and September and the dry season lasts from October to April. Wuchale district is located in $38^{\circ} 47^{\prime} \mathrm{E}$ longitude and $9^{\circ} 54^{\prime} \mathrm{N}$ latitude with maximum temperature of $25^{\circ} \mathrm{C}$ and minimum of $3^{\circ} \mathrm{C}$. Similarly, the main rainy season of Wuchale district occurs between May and September and the dry season lasts from October to April. Clay and sandy soils are the major soil types of the zone. In both of the districts agricultural production is characterized by a mixed crop-livestock production system [9] (Figure 1).

*Corresponding author: Teka Feyera, Jigjiga University College of Veterinary Medicine, Ethiopia, Tel: +251913199648; E-mail: teka841@yahoo.com

Received April 12, 2014; Accepted May 31, 2014; Published June 10, 2014

Citation: Abera B, Kebede K, Gizaw S, Feyera T (2014) On-Farm Phenotypic Characterization of Indigenous Sheep Types in Selale Area, Central Ethiopia. J Veterinar Sci Technol 5: 180. doi:10.4172/2157-7579.1000180

Copyright: (๑) 2014 Abera B, et al. This is an open-access article distributed under the terms of the Creative Commons Attribution License, which permits unrestricted use, distribution, and reproduction in any medium, provided the original author and source are credited. 


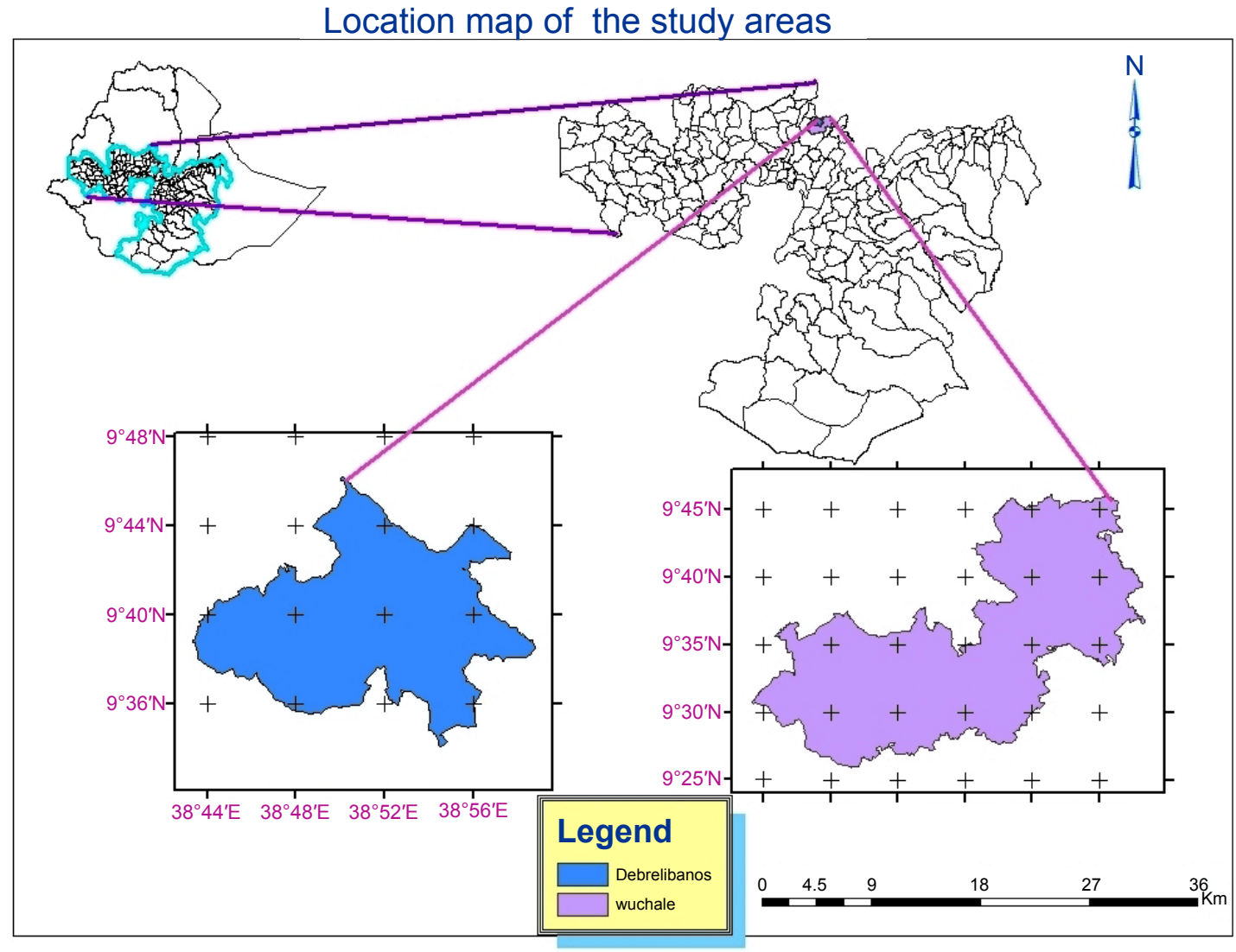

Figure 1: Location map of the study area.

\section{Sampling procedure}

Selection of the studied districts and peasant associations were done using multi-stage purposive sampling technique in consultation with zonal and district bureau of agriculture experts. Four Kebeles in Debre Libanos (Wakene, Sone, Dire Jibbo and Tere) and four Kebeles in Wuchale district (Jate, Harkiso, Adere Gordoma and Gora Keteba) were selected based on their suitability for sheep production, road access and willingness of the farmers to participate in the study. A total of 400 female (200 in Debre Libanos and 200 in Wuchale) and 160 male (80 in Debre Libanos and 80 in Wuchale) sheep were selected for body linear measurements.

\section{Data collection}

The standard breed descriptor list for sheep developed by FAO [10] was closely followed in selecting morphological variables. Qualitative traits like: coat color pattern, coat color type, hair type, head profile, ears, wattle, horn, ruff and tail were observed and recorded. Body measurements: Chest Girth (CG), Body Length (BL), Wither Height (WH), Rump width (RW), Ear Length (EL), Horn Length (HL), Tail Length (TL), Tail width (TW), Rump length (RL) and Scrotum circumference (SC) were measured using flexible measuring tape while weight was measured using suspended spring balance having $50 \mathrm{~kg}$ capacity with $0.2 \mathrm{~kg}$ precision. Each experimental animal was identified by sex, districts and age group. Adult sheep were classified into three age groups; 0PPI (zero pair of permanent incisor), 1PPI (one pair of permanent incisor) and 2PPI (two pair of permanent incisor. Linear body measurements were taken by restraining and holding the animals in a stable condition.

\section{Data management and analysis}

Statistical analyses were made separately for male and female animals on variables that varied on sex; otherwise the data were merged and analyzed together. Qualitative data from individual observation were analyzed following the frequency procedures of SAS version 9.1(2005). The General Linear Model (GLM) procedure of SAS was employed to analyze quantitative variables to determine effects of class variables (sex, district and dentition). The effects of class variables and their interaction were expressed as Least Square Means (LSM) \pm SE. Due to the low number of males in each dentition class, analysis was done for both sexes independently. Within each sex, location and dentition were fitted as fixed factors. Pearson's correlation coefficient between linear bodies measurements under consideration were computed for both of the sheep types within each sex.

\section{Univariate and multivariate analysis}

General linear model procedures (PROC GLM) of the SAS were employed for quantitative variables to detect statistical differences among sample sheep populations. Mean comparisons were made for variables showing significant differences between sample populations. The quantitative variables from female and male animals were separately subjected to discriminant analysis (PROC DISCRIM of SAS) and canonical discriminant analysis (CAN DISC) programme to ascertain the existence of population level phenotypic differences among the sample sheep populations in the study area. 
Citation: Abera B, Kebede K, Gizaw S, Feyera T (2014) On-Farm Phenotypic Characterization of Indigenous Sheep Types in Selale Area, Central Ethiopia. J Veterinar Sci Technol 5: 180. doi:10.4172/2157-7579.1000180

Page 3 of 6

\section{Results and Discussions}

\section{Characterization of qualitative traits}

The major qualitative traits of sample sheep population are presented in Table 1. Out of the sampled 390 sample sheep, $58.21 \%$ were plain, $33.33 \%$ patchy and $8.46 \%$ had spotted coat pattern. Brown and white with brown dominant $(24.87 \%)$, brown with red dominant (16.92\%), and white with black dominant (19.23\%) coat color patterns were the dominant colors. Beside, brown (18.21\%), black (4.36\%), white $(4.87 \%)$ and red $(6.67 \%)$ coat were also observed in plain pattern and mixed in patchy or spotted patterns.
Majority of sheep population $(80.77 \%)$ didn't have toggle and majority $(82.05 \%)$ lack ruff. Ear formation of sheep population was carried semi-pendulous $(86.67 \%)$ followed by rudimentary $(13.33 \%)$. Females were usually polled (74.84\%) with $48.72 \%$ straight concerning horn shape. Males were characterized by curved horn shape $(74.29 \%)$ and spiral horn shape (25.71\%) in Debre Libanos while 52\% and 48\% in Wuchale district, respectively. In $67.10 \%$ of the female population the tail was straight down pointed while the rest $32.90 \%$ had twisted tail. In contrary to the female sheep population male had straight with twisted end $(65.71 \%)$ while the rest $(34.29 \%)$ straight with tip down ward.

Majority of the sheep $(59.74 \%)$ in Wuchale and Debre Libanos

\begin{tabular}{|c|c|c|c|c|c|c|c|c|c|c|c|c|c|}
\hline \multirow{4}{*}{ Character } & \multirow{4}{*}{\multicolumn{3}{|c|}{ Attributes }} & \multicolumn{4}{|c|}{ Debre Libanos } & \multicolumn{4}{|c|}{ Wuchale } & \multirow{2}{*}{\multicolumn{2}{|c|}{ Overall }} \\
\hline & & & & \multicolumn{4}{|c|}{ Sex } & \multicolumn{4}{|c|}{ Sex } & & \\
\hline & & & & \multicolumn{2}{|c|}{ Female } & \multicolumn{2}{|c|}{ Male } & \multicolumn{2}{|l|}{ Female } & \multicolumn{2}{|c|}{ Male } & \\
\hline & & & & $\mathrm{N}$ & $\%$ & $\mathrm{~N}$ & $\%$ & $\mathrm{~N}$ & $\%$ & & $\%$ & & V \% \\
\hline \multirow{4}{*}{$\begin{array}{l}\text { Coat colour } \\
\text { pattern }\end{array}$} & \multicolumn{3}{|l|}{ Plain } & $89^{*}$ & 57.42 & $21^{*}$ & 60.00 & $97^{*}$ & 64.6 & & 40.00 & \multicolumn{2}{|c|}{22758.21} \\
\hline & \multicolumn{3}{|l|}{ Patchy } & 60 & 38.71 & 6 & 17.14 & 44 & 29.3 & & 40.00 & & 33.33 \\
\hline & \multicolumn{3}{|l|}{ Spotted } & 6 & 3.87 & 8 & 22.86 & 9 & 6.0 & & 20.00 & & 8.46 \\
\hline & \multicolumn{3}{|l|}{$X^{2}$-value } & \multicolumn{2}{|c|}{68.68} & \multicolumn{2}{|c|}{11.37} & \multicolumn{3}{|l|}{78.52} & $4.00 n s$ & & \\
\hline & White & & & $7^{*}$ & 4.52 & - & - & $6^{*}$ & 4.0 & & 12.00 & & 4.87 \\
\hline Coat colour type & Black & & & 10 & 6.45 & - & - & 7 & 4.6 & & - & & 4.36 \\
\hline & Brown & & & 23 & 14.84 & 8 & 22.86 & 32 & 21. & & 16.00 & & 18.21 \\
\hline & Red & & & 6 & 3.87 & 5 & 14.29 & 9 & 6.0 & & 12.00 & & 6.67 \\
\hline & white and red with white dor & ant & & 5 & 3.23 & - & - & 6 & 4.0 & & 16.00 & & 4.87 \\
\hline & Brown and White with browr & ominar & & 43 & 27.74 & 6 & 17.14 & 40 & 26. & & 16.00 & & 24.87 \\
\hline & Brown and red with red dom & & & 25 & 16.13 & 10 & 28.57 & 17 & 11. & & 28.00 & & 16.92 \\
\hline & Black and white with black $\mathrm{d}$ & ninant & & 36 & 23.23 & 6 & 17.14 & 33 & 22. & & - & 75 & 19.23 \\
\hline & $X^{2}$-value & & & & 1.72 & 2.2 & & 74.21 & & & $5.20 n s$ & & \\
\hline & Present & & & $44^{*}$ & 28.39 & - & - & $31^{*}$ & 20. & & - & & 19.23 \\
\hline loggle & Absent & & & 111 & 71.61 & 35 & 100 & 119 & 79. & & 100 & & 80.77 \\
\hline & $X^{2}$-value & & & & 8.96 & - & & 51.63 & & - & & & \\
\hline Character & & & & re Lib & banos & & & Wucl & chale & & & & \\
\hline & Attrih & & & Sex & & & & $\mathrm{Se}$ & ex & & & & rall \\
\hline & 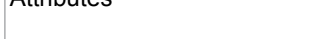 & & & & & & & nale & & & & & \\
\hline & & $\mathrm{N}$ & $\%$ & & $\mathrm{~N}$ & $\%$ & $\mathrm{~N}$ & $\%$ & & $\mathrm{~N}$ & $\%$ & $\mathrm{~N}$ & $\%$ \\
\hline & Long fat tail & $44^{*}$ & 92.90 & & $30^{*}$ & 85.71 & 141 & 94.0 & & 50 & 100 & 365 & 93.59 \\
\hline Tail type & Long thin tail & 11 & 7.10 & & 5 & 14.29 & 9 & 6.00 & & - & - & 25 & 6.41 \\
\hline & $X^{2}$-value & & & & & & & 16.6 & & & - & & \\
\hline & Straight and tip down ward & $104^{*}$ & 67.10 & & 12 & 34.29 & 81 & 54.00 & & $36^{*}$ & 72.00 & 233 & 59.74 \\
\hline Tail form & Straight and twisted end & 51 & 32.90 & & 23 & 65.71 & 69 & 46.00 & & 14 & 28.00 & 157 & 40.26 \\
\hline & $X^{2}$-value & & & & & 6ns & & 0.96 & & & 68 & & \\
\hline Ruff & Present & - & - & & $26^{*}$ & 74.29 & - & - & & $44^{*}$ & 88.00 & 70 & 17.95 \\
\hline & Absent & 155 & 100 & & 9 & 25.71 & 150 & 100 & & 6 & 12.00 & 320 & 82.05 \\
\hline & $X^{2}$-value & & & & & 26 & & - & & & 3.88 & & \\
\hline Ear form & Rudimentary & $24^{*}$ & 15.48 & & 5 & 14.29 & $15^{\star}$ & 10 & & $8^{*}$ & 16 & 52 & 13.33 \\
\hline & Semi-pendulous & 131 & 84.52 & & 30 & 85.71 & 135 & 90 & & 42 & 84 & 338 & 86.67 \\
\hline & $X^{2}$-value & & & & & 86 & & 6.00 & & & 312 & & \\
\hline & Straight & 17 & 48.72 & & - & - & 11 & 44.00 & & - & - & 28 & 19.72 \\
\hline Horn shane & Curved & 7 & 30.77 & & 26 & 74.29 & 6 & 24.00 & & 26 & 52.00 & 65 & 45.77 \\
\hline . & Spiral & & & & 925. & & 8 & 32.00 & & 24 & 48.00 & 49 & \\
\hline & $X^{2}$-value & & & & & & & $52 n s$ & & & $8 n s$ & & \\
\hline
\end{tabular}

$\mathrm{N}=$ number of households; Ns=non-significant; ${ }^{*} \mathrm{P}<0.05$

Table 1: Summary of the qualitative traits of female and male sheep in the study areas. 
districts had tails hanging straight downwards and $40.26 \%$ of them had twisted end. The higher proportion of male sheep with long and straight downward pointed tail might be due to selection against twisted end tail animals and farmers preferences of straight downward pointed tail. It was noted that ruff was mainly sex and age dependent. They were totally absent in females and more readily observed in adult males as compared to young growing males. The chi-square testfor assumption of equal proportion of categorical variables in both Wuchale and Debre Libanos sample sheep population indicated that among the variables considered in this study coat pattern, coat color, toggle, tail type, tail conformation and ear form were found to significantly $(\mathrm{P}<0.05)$ differ within the sample sheep population.

\section{Live body weight and linear measurements}

The body weight and linear measurements for Debre Libanos and Wuchale sheep population at various ages are presented in Table 2. Least squares means of most of the quantitative variables were significant $(\mathrm{P}<0.05)$ between districts except body weight and Scrotal circumferences (Table 2). Results for body weight and linear measurements of Debre Libanos and Wuchale sheep revealed that Debre Libanos had significantly larger $(\mathrm{P}<0.05)$ linear measurements than Wuchale sheep population.

Sex by age group: The interaction between sex and age group not significantly $(\mathrm{p}>0.05)$ affect ear length of the sheep. The remaining parameters of body measurements were affected by the sex-age interaction effect. Both females and males in age group 0(OPPI) had the same ( $p>0.05$ ) body weight value but males in age group 1(1PPI) and $\geq$ 2PPI were heavier $(\mathrm{p}<0.05)$ than females in the same age group. Body weight of males in age group 0PPI $(25.7 \pm 0.3 \mathrm{~kg})$, age group 1PPI (31.9 $\pm 0.8 \mathrm{~kg})$ and age group $\geq 2 \mathrm{PPI}(38.2 \pm 2.0 \mathrm{~kg})$ in the current study was higher than body weight of Menz males18.0 $\pm 0.28 \mathrm{~kg},(22.9 \pm 0.39 \mathrm{~kg})$ and $24.9 \pm 0.67$ in the same age group. Similarly, body weight of females in all age group in the current study was higher than the values reported for Menz ewes $(19.1 \pm 0.27 \mathrm{~kg})$ in the same age group [11].

\begin{tabular}{|c|c|c|c|c|c|c|c|c|c|c|c|}
\hline \multirow{2}{*}{ Effects and level } & \multirow{2}{*}{$\mathbf{N}$} & BW & CG & $\mathrm{BL}$ & WH & RW & EL & TL & TW & $\mathrm{RL}$ & SC \\
\hline & & $\mathrm{LSM} \pm \mathrm{SE}$ & LSM \pm SE & $\mathrm{LSM} \pm \mathrm{SE}$ & $\mathrm{LSM} \pm \mathrm{SE}$ & LSM \pm SE & LSM \pm SE & $\mathrm{LSM} \pm \mathrm{SE}$ & $\mathrm{LSM} \pm \mathrm{SE}$ & $\mathrm{LSM} \pm \mathrm{SE}$ & $\mathrm{LSM} \pm \mathrm{SE}$ \\
\hline Over all & 560 & $27.75 \pm 0.2$ & $75.34 \pm 0.2$ & $62.59 \pm 0.2$ & $65.43 \pm 0.1$ & $16.70 \pm 0.1$ & $9.49 \pm 0.1$ & $32.31 \pm 0.2$ & $11.38 \pm 0.1$ & $21.08 \pm 0.1$ & $21.90 \pm 0.26$ \\
\hline CV\% & & 12.89 & 5.36 & 5.15 & 4.76 & 7.52 & 20.88 & 16.56 & 15.65 & 6.18 & 14.82 \\
\hline$R^{2}$ & & 0.27 & 0.33 & 0.24 & 0.21 & 0.19 & 0.02 & 0.07 & 0.51 & 0.12 & 0.015 \\
\hline Location & & NS & NS & * & * & * & * & NS & * & * & NS \\
\hline D/Libanos & 280 & $27.6 \pm 0.2$ & $75.05 \pm 0.3$ & $62.9 \pm 0.2^{\mathrm{a}}$ & $65.73 \pm 0.2^{a}$ & $16.78 \pm 0.1^{a}$ & $9.71 \pm 0.1^{a}$ & $32.99 \pm 0.3$ & $12.45 \pm 0.2^{a}$ & $21.38 \pm 0.1^{\mathrm{a}}$ & $21.50 \pm 0.38$ \\
\hline Wuchale & 280 & $27.9 \pm 0.2$ & $74.31 \pm 0.3$ & $62.3 \pm 0.2^{\mathrm{b}}$ & $64.86 \pm 0.2^{b}$ & $16.35 \pm 0.1^{b}$ & $9.28 \pm 0.1^{b}$ & $32.67 \pm 0.3$ & $11.85 \pm 0.1^{b}$ & $20.88 \pm 0.1^{b}$ & $22.30 \pm 0.35$ \\
\hline Sex & & NS & * & * & * & * & NS & * & * & NS & - \\
\hline Female & 400 & $27.96 \pm 0.2$ & $76.23 \pm 0.2^{a}$ & $63.51 \pm 0.2^{a}$ & $65.6 \pm 0.2^{\mathrm{a}}$ & $16.88 \pm 0.1^{a}$ & $9.50 \pm 0.1$ & $31.63 \pm 0.3^{a}$ & $10.33 \pm 0.1^{a}$ & $21.23 \pm 0.1$ & NA \\
\hline Male & 160 & $27.23 \pm 0.4$ & $73.13 \pm 0.5^{b}$ & $60.30 \pm 0.3^{b}$ & $65.0 \pm 0.3^{b}$ & $16.24 \pm 0.1^{b}$ & $9.48 \pm 0.1$ & $34.02 \pm 0.4^{b}$ & $14.03 \pm 0.2^{b}$ & $21.03 \pm 0.1$ & $21.90 \pm 0.26$ \\
\hline
\end{tabular}

a,b,c means on the same column with different superscripts within the specified dentition group are significantly different $(P<0.05)$; Ns $=N o n-s i g n i f i c a n t ~(P>0.05) ; B W=B o d y$ weight; $\mathrm{BL}=$ Body Length; $\mathrm{CG}=$ Chest Girth; $\mathrm{WH}=$ Wither height; $\mathrm{RW}=$ Rump width $; \mathrm{EL}=$ Ear Length; $\mathrm{TL}=\mathrm{Tail}$ Length; $\mathrm{TW}=\mathrm{Tail}$ width; $\mathrm{RL}=\mathrm{Rump}$ length; $\mathrm{SC}=\mathrm{Scrota}$ Circumference; D/Libanos =Debre Libanos.

\begin{tabular}{|c|c|c|c|c|c|c|c|c|c|c|c|}
\hline \multirow{2}{*}{ Effects and level } & \multirow{2}{*}{$\mathbf{N}$} & BW & CG & BL & WH & RW & EL & $\mathrm{TL}$ & TW & $\mathrm{RL}$ & SC \\
\hline & & LSM \pm SE & LSM \pm SE & LSM \pm SE & LSM \pm SE & LSM \pm SE & $\mathrm{LSM} \pm \mathrm{SE}$ & LSM \pm SE & LSM \pm SE & LSM \pm SE & LSM $\pm S E$ \\
\hline Age group & 560 & $*$ & * & * & * & * & Ns & $*$ & * & $*$ & * \\
\hline 0 PPI & 156 & $25.43 \pm 0.3^{c}$ & $71.50 \pm 0.4^{b}$ & $59.83 \pm 0.3^{c}$ & $63.59 \pm 0.3^{b}$ & $15.91 \pm 0.1^{b}$ & $9.32 \pm 0.1$ & $33.51 \pm 0.4^{a}$ & $12.77 \pm 0.2^{\mathrm{a}}$ & $20.78 \pm 0.1^{b}$ & $21.29 \pm 0.3^{b}$ \\
\hline $1 \mathrm{PPI}$ & 88 & $27.83 \pm 0.5^{\mathrm{b}}$ & $76.31 \pm 0.6^{a}$ & $62.89 \pm 0.4^{\mathrm{b}}$ & $66.26 \pm 0.4^{\mathrm{a}}$ & $17.01 \pm 0.2^{\mathrm{a}}$ & $9.42 \pm 0.2$ & $33.50 \pm 0.6^{a}$ & $12.25 \pm 0.4^{a}$ & $21.40 \pm 0.2^{\mathrm{a}}$ & $24.10 \pm 0.7^{\mathrm{a}}$ \\
\hline$\geq 2 \mathrm{PPI}$ & 316 & $28.87 \pm 0.2^{\mathrm{a}}$ & $76.97 \pm 0.2^{\mathrm{a}}$ & $63.87 \pm 0.2^{\mathrm{a}}$ & $66.12 \pm 0.2^{a}$ & $17.00 \pm 0.1^{a}$ & $9.60 \pm 0.1$ & $31.39 \pm 0.3^{b}$ & $10.45 \pm 0.1^{b}$ & $21.14 \pm 0.1^{a}$ & $24.60 \pm 1.3^{a}$ \\
\hline Sex by age group & 560 & * & * & * & * & * & NS & * & * & * & - \\
\hline Female, OPPI & 30 & $24.3 \pm 0.6^{d}$ & $73.13 \pm 0.7^{c}$ & $61.16 \pm 0.6^{b}$ & $62.63 \pm 0.6^{d}$ & $16.34 \pm 0.2^{c}$ & $8.97 \pm 0.4$ & $31.37 \pm 0.8^{\mathrm{bc}}$ & $10.13 \pm 0.2^{c}$ & $20.47 \pm 0.1^{d}$ & NA \\
\hline Male, 0 PPI & 126 & $25.7 \pm 0.3^{d}$ & $71.11 \pm 0.4^{d}$ & $59.52 \pm 0.3^{c}$ & $63.82 \pm 0.3^{d}$ & $15.80 \pm 0.1^{d}$ & $9.41 \pm 0.2$ & $34.03 \pm 0.5^{\mathrm{a}}$ & $13.40 \pm 0.2^{b}$ & $20.86 \pm 0.1^{\text {cd }}$ & $21.29 \pm 0.3^{b}$ \\
\hline Female, 1PPI & 59 & $25.81 \pm 0.5^{d}$ & $74.39 \pm 0.6^{c}$ & $62.94 \pm 0.5^{a}$ & $65.00 \pm 0.4^{c}$ & $16.64 \pm 0.1^{\mathrm{bc}}$ & $9.27 \pm 0.3$ & $33.18 \pm 0.6^{\mathrm{ab}}$ & $10.34 \pm 0.2^{c}$ & $20.94 \pm 0.2^{\text {cd }}$ & NA \\
\hline Male, 1 PPI & 29 & $31.93 \pm 0.8^{b}$ & $80.24 \pm 0.7^{a}$ & $62.79 \pm 0.7^{\mathrm{ab}}$ & $68.83 \pm 0.6^{a}$ & $17.75 \pm 0.3^{a}$ & $9.72 \pm 0.3$ & $34.14 \pm 1.1^{a}$ & $16.14 \pm 0.6^{a}$ & $22.34 \pm 0.4^{b}$ & $24.10 \pm 0.7^{a}$ \\
\hline Female, $\geq 2$ PPI & 311 & $28.7 \pm 0.2^{c}$ & $76.88 \pm 0.2^{b}$ & $63.85 \pm 0.2^{\mathrm{a}}$ & $66.03 \pm 0.2^{b}$ & $16.97 \pm 0.0^{\mathrm{b}}$ & $9.59 \pm 0.1$ & $31.36 \pm 0.3^{b}$ & $10.34 \pm 0.1^{c}$ & $21.09 \pm 0.1^{c}$ & NA \\
\hline Male , $\geq 2$ PPI & 5 & $38.2 \pm 2.0^{\mathrm{a}}$ & $82.60 \pm 1.6^{a}$ & $65.40 \pm 1.7^{a}$ & $71.60 \pm 2.2^{\mathrm{a}}$ & $18.60 \pm 0.7^{a}$ & $10.00 \pm 0.0$ & $33.20 \pm 2.6^{\mathrm{ab}}$ & $17.40 \pm 0.8^{a}$ & $24.00 \pm 1.6^{a}$ & $24.60 \pm 1.3^{a}$ \\
\hline
\end{tabular}

a,b,c,d means on the same column with different superscripts within the specified dentition group are significantly different $(P<0.05)$; Ns $=N$ Non- significant $(P>0.05) ; B W=$ Body weight; $B L=$ Body Length; $C G=$ Chest Girth; $W H=$ Wither height; $R W=$ Rump width $; E L=E a r ~ L e n g t h ; T L=$ Tail Length; $T W=$ Tail width; RL= Rump length; SC = Scrotal Circumference; OPPI = 0 Pair of Permanent Incisors; 1 PPI = 1 Pair of Permanent Incisors; 2 PPI = 2 Pairs of Permanent Incisors; NA=Not applicable

\begin{tabular}{|c|c|c|c|c|c|c|c|c|c|c|c|}
\hline \multirow{2}{*}{ Effects and level } & \multirow{2}{*}{$\mathbf{N}$} & BW & CG & $\mathrm{BL}$ & WH & RW & EL & TL & TW & $\mathrm{RL}$ & SC \\
\hline & & LSM \pm SE & $\mathrm{LSM} \pm \mathrm{SE}$ & LSM $\pm S E$ & LSM \pm SE & LSM \pm SE & LSM \pm SE & $\mathrm{LSM} \pm \mathrm{SE}$ & LSM \pm SE & LSM \pm SE & LSM \pm SE \\
\hline Age by location & 560 & * & * & $*$ & * & * & * & $*$ & * & * & * \\
\hline OPPI, D/Libanos & 70 & $25.11 \pm 0.4^{c}$ & $71.03 \pm 0.5^{c}$ & $59.85 \pm 0.4^{c}$ & $63.57 \pm 0.4^{c}$ & $16.02 \pm 0.2^{c}$ & $9.41 \pm 0.2^{\mathrm{ab}}$ & $34.03 \pm 0.6^{\mathrm{a}}$ & $13.12 \pm 0.3^{a}$ & $20.98 \pm 0.2^{c}$ & $20.57 \pm 0.38^{c}$ \\
\hline 0 PPI,Wuchale & 86 & $25.69 \pm 0.4^{c}$ & $71.89 \pm 0.5^{c}$ & $59.82 \pm 0.3^{c}$ & $63.61 \pm 0.4^{c}$ & $15.81 \pm 0.1^{c}$ & $9.26 \pm 0.2^{b}$ & $33.11 \pm 0.6^{\mathrm{ab}}$ & $12.48 \pm 0.2^{\mathrm{ab}}$ & $20.62 \pm 0.2^{c}$ & $21.93 \pm 0.34^{b}$ \\
\hline 1PPI, D/Libanos & 42 & $26.73 \pm 0.7^{b}$ & $75.95 \pm 0.8^{b}$ & $62.67 \pm 0.5^{b}$ & $66.57 \pm 0.5^{a}$ & $17.23 \pm 0.2^{\mathrm{ab}}$ & $9.59 \pm 0.3^{a b}$ & $32.81 \pm 0.9^{\mathrm{ab}}$ & $12.67 \pm 0.6^{\mathrm{ab}}$ & $21.79 \pm 0.3^{a}$ & $24.20 \pm 0.8^{a}$ \\
\hline 1PPI, Wuchale & 46 & $28.82 \pm 0.7^{a}$ & $76.65 \pm 0.7^{a b}$ & $63.11 \pm 0.6^{b}$ & $65.97 \pm 0.5^{\mathrm{ab}}$ & $16.74 \pm 0.2^{\mathrm{ab}}$ & $9.26 \pm 0.3^{\mathrm{ab}}$ & $34.13 \pm 0.7^{a}$ & $11.87 \pm 0.5^{b}$ & $21.06 \pm 0.2^{b c}$ & $24.00 \pm 1.1^{\mathrm{a}}$ \\
\hline$\geq 2 \mathrm{PPI}, \mathrm{D} /$ Libanos & 168 & $28.91 \pm 0.3^{a}$ & $77.58 \pm 0.5^{a}$ & $64.29 \pm 0.2^{\mathrm{a}}$ & $66.92 \pm 0.2^{a}$ & $17.16 \pm 0.1^{a}$ & $9.87 \pm 0.2^{\mathrm{a}}$ & $31.99 \pm 0.5^{b}$ & $10.74 \pm 0.1^{c}$ & $21.32 \pm 0.1^{\mathrm{ab}}$ & $24.60 \pm 1.33^{a}$ \\
\hline$\geq 2$ PPI,Wuchale & 148 & $28.83 \pm 0.3^{a}$ & $76.28 \pm 0.3^{b}$ & $63.40 \pm 0.3^{b}$ & $65.20 \pm 0.2^{b}$ & $16.86 \pm 0.1^{b}$ & $9.30 \pm 0.2^{b}$ & $30.71 \pm 0.4^{c}$ & $10.12 \pm 0.1^{d}$ & $20.94 \pm 0.1^{c}$ & NA \\
\hline
\end{tabular}

Table 2: Least squares Means ( \pm S.E.) for Body Weight $(\mathrm{kg})$, and Linear body measurements $(\mathrm{cm})$ as affected by district, sex, age group and their interactions. 
Citation: Abera B, Kebede K, Gizaw S, Feyera T (2014) On-Farm Phenotypic Characterization of Indigenous Sheep Types in Selale Area, Central Ethiopia. J Veterinar Sci Technol 5: 180. doi:10.4172/2157-7579.1000180

Page 5 of 6

Age by location:The interaction of age group and location was significant $(\mathrm{p}<0.05)$ for all parameters of body measurement used in the study. The result indicates the interaction of age group and location had strong effect on body weight and linear body measurements. Young (0PPI) sheep of DebreLibanos had similar ( $p>0.05)$ body weight with Wuchale sheep. The age group $\geq 2$ PPI also had similar $(\mathrm{p}>0.05)$ body weight 2 locations. However, Wuchale sheep $(28.8 \pm 0.7 \mathrm{~kg})$ in age group 1PPI had heavier body weight than Debre Libanos $(26.7 \pm 0.7$ $\mathrm{kg}$ ). This implies that Debre Libanos sheep did not attain maturity at 2PPI. Body weight of the age group 1PPI of the two locations is larger than Tocha, Mareka and Konta sheep [12] and Menz sheep [11] in the same age groups.

\section{Correlation between body weight and body measurements}

The highest relationship between chest girth and body weight were observed in Debre Libanos female sheep (0.68) and in Wuchale male sheep (0.80).The highest correlation of chest girth with body weight than other body measurements was in harmony with other results of
[11,13-15] and it can indicate that chest girth is the best variable for predicting live weight than other measurements (Table 3 ).

Of the linear body measurements, chest girth with exception in Wuchale females had the highest correlation with body weight at all groups. In both Wuchale and Debre Libanos districts, the highest correlation coefficient between body weight andchest girth, body length, wither height and rump height were established in males $(73 \%$, $53 \%, 62 \%, 64 \%)$ for Debre Libanos and $(80 \%, 73 \%, 72 \%, 75 \%)$ for Wuchale. The highest positive and significant correlation between body weight and chest girthsuggest thatthis variables couldprovide a good estimate for predicting live weight of these breed types (Table 4).

\section{Multiple regression analysis}

Table 5 shows that the number of variables entered in the model to predict the best fitted variable to estimate body weight and their contribution in terms of adjusted coefficient of determination ( $\mathrm{R}^{2}$ adj.), mallows Cp statistics, Akaike Information Criterion (AIC)

\begin{tabular}{|c|c|c|c|c|c|c|c|c|c|}
\hline & CG & $B L$ & $\mathrm{WH}$ & $\mathrm{RH}$ & RW & TL & TW & $\mathrm{RL}$ & BW \\
\hline CG & & $0.39^{*}$ & $0.33^{*}$ & $0.42^{*}$ & $0.20^{*}$ & $0.13^{\text {ns }}$ & $0.08^{\text {ns }}$ & $0.18^{*}$ & $0.68^{*}$ \\
\hline $\mathrm{BL}$ & $0.68^{*}$ & & $0.29^{*}$ & $0.30^{*}$ & $0.08^{\text {ns }}$ & $0.11^{\mathrm{ns}}$ & $-0.06^{n s}$ & $0.08^{\text {ns }}$ & $0.38^{*}$ \\
\hline $\mathrm{WH}$ & $0.66^{*}$ & $0.57^{*}$ & & $0.84^{*}$ & $0.13^{\text {ns }}$ & $0.07^{\text {ns }}$ & $0.10^{\text {ns }}$ & $0.12^{\text {ns }}$ & $0.30^{*}$ \\
\hline $\mathrm{RH}$ & $0.68^{*}$ & $0.56^{*}$ & $0.91^{*}$ & & $0.15^{\star}$ & $0.07^{\mathrm{ns}}$ & $0.06^{\text {ns }}$ & $0.15^{\star}$ & $0.37^{*}$ \\
\hline RW & $0.60^{*}$ & $0.43^{*}$ & $0.40^{*}$ & $0.39^{\star}$ & & $0.08^{\text {ns }}$ & $0.40^{\star}$ & $0.11^{\mathrm{ns}}$ & $0.23^{*}$ \\
\hline $\mathrm{TL}$ & $-0.23^{*}$ & $-0.08^{n s}$ & $-0.23^{*}$ & $-0.30^{*}$ & $-0.15^{\text {ns }}$ & & $0.19^{*}$ & $0.15^{*}$ & $0.22^{*}$ \\
\hline TW & $0.64^{*}$ & $0.33^{*}$ & $0.33^{*}$ & $0.35^{\star}$ & $0.54^{*}$ & $-0.09^{n s}$ & & $0.15^{\star}$ & $0.22^{*}$ \\
\hline $\mathrm{RL}$ & $0.59^{*}$ & $0.41^{*}$ & $0.46^{*}$ & $0.51^{*}$ & $0.39^{\star}$ & $-0.25^{\star}$ & $0.22^{\text {ns }}$ & & $0.26^{*}$ \\
\hline BW & $0.73^{*}$ & $0.53^{*}$ & $0.62^{*}$ & $0.64^{*}$ & $0.42^{*}$ & $-0.14^{\mathrm{ns}}$ & $0.44^{\star}$ & $0.53^{*}$ & \\
\hline SC & $0.50^{*}$ & $0.43^{*}$ & $0.33^{*}$ & $0.37^{*}$ & 0.37 & $-0.11^{\mathrm{ns}}$ & $0.40^{*}$ & $0.13^{\text {ns }}$ & $0.37^{*}$ \\
\hline
\end{tabular}

NS= Non-significant $(\mathrm{P}<0.05)$; significant at 0.05 level; BL=Body Length; $C G=$ Chest Girth; WH=Wither height; RH=Rump Height; RW =Rump Width; TL=Tail Length; TW=Tail Width RL=Rump Length; BW=Body Weight; SC =Scrotal circumference

Table 3: Correlation coefficients among body measurements and weight of females and males of indigenous Debre Libanos sheep (values above the diagonal are for females and below the diagonal are for males) ( $\mathrm{N}=80$ for male; $\mathrm{N}=200$ for females).

\begin{tabular}{|c|c|c|c|c|c|c|c|c|c|c|}
\hline & CG & $\mathrm{BL}$ & $\mathrm{WH}$ & $\mathrm{RH}$ & $\mathrm{RW}$ & $\mathrm{TL}$ & TW & $\mathrm{RL}$ & BW & SC \\
\hline CG & & $0.71^{*}$ & $0.75^{\star}$ & $0.75^{*}$ & $0.51^{*}$ & $0.31^{*}$ & $0.41^{*}$ & $0.51^{*}$ & $0.80^{*}$ & $0.32^{*}$ \\
\hline $\mathrm{BL}$ & $0.57^{\star}$ & & $0.71^{\star}$ & $0.75^{*}$ & $0.42^{*}$ & $0.39^{*}$ & $0.43^{*}$ & $0.49^{*}$ & $0.73^{\star}$ & $0.23^{*}$ \\
\hline WH & $0.26^{\star}$ & $0.36^{*}$ & & $0.95^{*}$ & $0.42^{*}$ & $0.42^{*}$ & $0.43^{*}$ & $0.53^{*}$ & $0.72^{*}$ & $0.23^{*}$ \\
\hline $\mathrm{RH}$ & $0.28^{*}$ & $0.39^{*}$ & $0.90^{*}$ & & $0.42^{*}$ & $0.46^{*}$ & $0.44^{*}$ & $0.53^{*}$ & $0.75^{\star}$ & $0.28^{*}$ \\
\hline RW & $0.33^{*}$ & $0.19^{*}$ & $0.26^{\star}$ & $0.28^{*}$ & & $0.08^{\mathrm{Ns}}$ & $0.57^{\star}$ & $0.45^{*}$ & $0.52^{*}$ & $0.16^{\mathrm{Ns}}$ \\
\hline $\mathrm{TL}$ & $0.07 \mathrm{Ns}$ & $0.17^{*}$ & $0.18^{*}$ & $0.19^{*}$ & $-0.08^{\mathrm{Ns}}$ & & $0.09^{\mathrm{Ns}}$ & $0.38^{*}$ & $0.35^{\star}$ & $0.17^{\mathrm{Ns}}$ \\
\hline TW & $0.17^{\star}$ & $0.26^{*}$ & $0.16^{\star}$ & $0.21^{*}$ & $0.09^{\mathrm{Ns}}$ & $0.03^{\mathrm{Ns}}$ & & $0.34^{*}$ & $0.51^{\star}$ & $0.23^{*}$ \\
\hline $\mathrm{RL}$ & $0.34^{*}$ & $0.28^{*}$ & $0.27^{\star}$ & $0.31^{*}$ & $0.43^{*}$ & $0.07^{\mathrm{Ns}}$ & $0.20^{\star}$ & & $0.48^{*}$ & $0.13^{\mathrm{Ns}}$ \\
\hline BW & $0.50^{\star}$ & $0.48^{*}$ & $0.32^{*}$ & $0.33^{*}$ & $0.24^{*}$ & $0.11^{\mathrm{Ns}}$ & $0.11^{\mathrm{Ns}}$ & $0.18^{*}$ & & $0.30^{\star}$ \\
\hline
\end{tabular}

$\mathrm{NS}=$ Non-significant $(\mathrm{P}<0.05) ;{ }^{*}$ significant at 0.05 level; BL=Body Length; $C G=$ Chest Girth; WH=Wither height; RH=Rump Height; RW =Rump Width; TL=Tail Length; TW=Tail Width RL=Rump Length; BW=Body Weight; SC =Scrotal circumference

Table 4: Correlation coefficients among body measurements and weight of females and males of Wuchale sheep (values above the diagonal are for males and below the diagonal are for females) ( $\mathrm{N}=80$ for male; $\mathrm{N}=200$ for females).

\begin{tabular}{|c|c|c|c|c|c|c|c|}
\hline \multirow[b]{2}{*}{ Age group } & \multirow[b]{2}{*}{ Model } & \multirow[b]{2}{*}{ Intercept } & \multicolumn{5}{|c|}{ Parameters } \\
\hline & & & $\beta_{1}$ & $\beta_{2}$ & $\beta_{3}$ & $\beta_{4}$ & $\mathrm{R}^{2}$ adjust. \\
\hline \multicolumn{8}{|l|}{ Female } \\
\hline OPPI & $\mathrm{CG}+\mathrm{BL}+\mathrm{WH}+\mathrm{RW}$ & $-53.95 \pm 6.96$ & $0.44 \pm 0.08$ & $0.31 \pm 0.10$ & $0.55 \pm 0.10$ & $-0.48 \pm 0.25$ & 0.83 \\
\hline 1PPI & CG & $-0.32 \pm 7.68$ & $0.35 \pm 0.10$ & & & & 0.16 \\
\hline$\geq 2 \mathrm{PPI}$ & $\mathrm{CG}+\mathrm{BL}$ & $-15.12 \pm 3.9$ & $0.41 \pm 0.05$ & $0.18 \pm 0.06$ & & & 0.30 \\
\hline Overall & $\mathrm{CG}+\mathrm{BL}+\mathrm{WH}$ & $-23.49 \pm 4.00$ & $0.44 \pm 0.04$ & $0.16 \pm 0.05$ & $0.11 \pm 0.05$ & & 0.36 \\
\hline \multicolumn{8}{|l|}{ Male } \\
\hline OPPI & $\mathrm{CG}+\mathrm{BL}+\mathrm{WH}$ & $-26.25 \pm 4.49$ & $0.38 \pm 0.06$ & $0.23 \pm 0.10$ & $0.18 \pm 0.08$ & & 0.57 \\
\hline$\geq 1 \mathrm{PPI}$ & $\mathrm{CG}+\mathrm{RH}$ & $-61.74 \pm 20.94$ & $0.52 \pm 0.19$ & $0.72 \pm 0.23$ & & & 0.36 \\
\hline Overall & $\mathrm{CG}+\mathrm{BL}+\mathrm{RH}$ & $-35.83 \pm 4.27$ & $0.43 \pm 0.06$ & $0.15 \pm 0.09$ & $0.33 \pm 0.08$ & & 0.67 \\
\hline
\end{tabular}

NB: It is difficult to put the value of AIC, Cp and BIC although the above table was estimated based on the value of AIC, Cp and BIC.

Table 5: Multiple regression analysis of live weight on different body measurements for ewe and ram by age group. 
Citation: Abera B, Kebede K, Gizaw S, Feyera T (2014) On-Farm Phenotypic Characterization of Indigenous Sheep Types in Selale Area, Central Ethiopia. J Veterinar Sci Technol 5: 180. doi:10.4172/2157-7579.1000180

Page 6 of 6

and Bayesian Information Criterion (BIC) at different dentition and sex categories.

Small AIC, Cp and BIC value and higher adjusted $\mathrm{R}^{2}$ are included in regression equation. The independent variables were body length, chest girth; wither height, rump height, rump width, tail length, tail width and rump length. In addition to these variables scrotal circumference and horn length were considered for male population.In most cases heart girth was found to be the most important in accounting sizeable proportion of the changes in the body weight. Similarly, this measurement was reported for Afar, Menz, Bonga, Horro, Gumuz, Jarso, and Nedjo sheep [11,16-18]. Chest girth was more reliable in predicting body weight than other linear body measurements. Parameter estimates in multiple linear regression model showed that higher $\mathrm{R}^{2}$ adjusted was observed when more than one body dimensions were used in the multiple regression equation.

\section{Conclusion}

The present study conducted in Selale area of Central Ethiopia evidenced that most of the sheep in the study area had plain coat pattern, brown coat color, semi-pendulous ear orientation and long fat tailed with straight down pointed in females and twisted end in males. Sex of the sheep had no significant ( $\mathrm{p}>0.05)$ effect on the body weight, ear length and rump length. Age group had significant effect $(\mathrm{p}<0.05)$ on body weight and other body measurements. Location was found to influence $(\mathrm{P}<0.05)$ body length, tail width, wither height, ear length and rump length. The interaction of sex and age group significantly $(\mathrm{p}<0.05)$ influenced all linear body measurements except ear length of the sheep. The interaction of age group and location was significant $(\mathrm{p}<0.05)$ for all linear body measurements. Of the linear body measurements, chest girth with exception in Wuchale females had the highest correlation with body weight at all groups. In the regression analysis carried out to predict body weight, heart girth was selected and explained more variation than other variables in all age groups of both males and females of Debre Libanos and Wuchale sheep population. For any breed improvement program and to boost productivity of indigenous sheep, characterization is the baseline. Therefore, this preliminary work could be used to support genetic analyses to determine variation between and within these small populations.

\section{References}

1. Roessler R, Drucker AG, Scarpa R, Markemann A, Lemke U, et al. (2008) Using choice experiments to assess smallholder farmers' preferences for pig breeding traits in different production systems in North-West Vietnam. Ecological Economics, 66: 184-192.

2. Duguma G, Mirkena T, Haile A, Iñiguez L, Okeyo AM (2010) Participatory approaches to investigate breeding objectives of livestock keepers. Livestock Research for Rural Development. Volume 22.

3. Siegmund-Schultze M, Legesse G, Abebe G, Valle Zárate A (2009) Bottleneck analysis of sheep production systems in southern Ethiopia: Comparison of reproductive and growth parameters. Options Mediterranean's, 91: 67-69.

4. Gizaw S1, Van Arendonk JA, Komen H, Windig JJ, Hanotte O (2007) Population structure, genetic variation and morphological diversity in indigenous sheep of Ethiopia. Anim Genet 38: 621-628.

5. CSA (Central Statistics Authority) (2010) Agricultural sample survey, 2009/10 (2002 E.C). Volume II, report on livestock and livestock characteristics. Addis Ababa, Ethiopia.
6. DAGRIS (2006) Domestic Animal Genetic Resources Information System (DAGRIS). International Livestock Research Institute, Addis Ababa, Ethiopia.

7. Workneh A (1992) Preliminary Survey of Indigenous Goat Types and Goat Husbandry Practices in Southern Ethiopia. MSc thesis, Alemaya University of Agriculture, Alemaya, Ethiopia. pp 153.

8. Rege JEO (2001) Defining livestock breeds in the context of community based management of farm animal genetic resources. In the Proceedings of Community based management of animal genetic resources held in Mbabane, Swaziland, pp: 27-35.

9. ZARDO (2010) Zonal agricultural development Office of North Shoa, Central Ethiopia.

10. FAO (2010) (Food and Agriculture Organization of United Nation). Sheep descriptors. In: Animal Genetic Resource Data Banks. Descriptor Lists for Cattle, Buffalo, Sheep and Goats. Animal Production and Health paper. Italy.

11. Tesfaye G (2008) Characterization of Menz and Afar Indigenous Sheep Breeds of Smallholders and Pastoralists for Designing Community-Based Breeding Strategies in Ethiopia, M.S. thesis, Haramaya Univ., Haramaya, Ethiopia.

12. Amelmal A (2011) Phenotypic Characterization of Indigenous Sheep Types of Dawuro Zone and Konta Special Woreda of SNNPR, M.S. thesis, Haramaya Univ, Haramaya, Ethiopia.

13. Thiruvenkadan AK, Karunanithi K, Murugan M, Arunachalam K, Narendra Babu R (2009) A comparative study on growth performance of crossbred and purebred Mecheri sheep raised under dry land farming conditions. South African Journal of Animal Science, 39: 121-125.

14. Fasae OA, Chineke AC, Alokan JA (2006) Relationship between some Physica Parameters of Grazing Yankasa Ewes in the Humid Zone of Nigeria. University of Agriculture. P.M.B. 2240. Abeokuta. Nigeria.

15. Solomon AG, Hegde BP, Taye M (2011) Growth and Physical Body Characteristics of Gumuz Sheep under Traditional Management Systems in Amhara Regional State, Ethiopia. Livestock Research for Rural Development 117: 23-27.

16. Zewdu E (2008) Characterization of Bonga and Horro Indigenous Sheep Breeds of Smallholders for Designing Community Based Breeding Strategies in Ethiopia, M.S. thesis submitted to the School of Graduate Studies of Haramaya Univ., Haramaya, Ethiopia.

17. Solomon A (2007) In-situ characterization of Gumuz sheep under farmers management in north-western lowlands of Amhara region. MSc. Thesis, Haramaya University, Ethiopia. pp 108.

18. Kedjela T (2010) On-Farm Phenotypic Characterization of Indigenous Sheep and Sheep Production Systems in West Wollega, Oromia, MS thesis Haramaya Univ, Ethiopia. 